\title{
Role of C-Arm VasoCT in the Use of Endovascular WEB Flow Disruption in Intracranial Aneurysm Treatment
}

\author{
J. Caroff, C. Mihalea, H. Neki, D. Ruijters, L. Ikka, N. Benachour, J. Moret, and L. Spelle
}

\begin{abstract}
BACKGROUND AND PURPOSE: The WEB aneurysm embolization system is still under evaluation but seems to be a promising technique to treat wide-neck bifurcation aneurysms. However, this device is barely visible using conventional DSA; thus, high-resolution contrastenhanced flat panel detector CT (VasoCT) may be useful before detachment to assess the sizing and positioning of the WEB. The purpose of this study was to evaluate the interest of VasoCT during WEB procedures.
\end{abstract}

MATERIALS AND METHODS: From March 2012 to July 2013, twelve patients (10 women and 2 men; age range, 44-55 years) were treated for 13 intracranial aneurysms with the WEB device. DSA and VasoCT were used and compared to depict any protrusion of the device in parent arteries before detachment. Two neuroradiologists reviewed each VasoCT scan, and the quality was graded on a subjective quality scale.

RESULTS: The mesh of the WEB was very well-depicted in all cases, allowing a very good assessment of its deployment. Device protrusion was clearly detected with VasoCT in 5 cases, leading to WEB repositioning or size substitution. During follow-up, VasoCT also allows good assessment of eventual residual blood flow inside the aneurysm or the WEB device.

CONCLUSIONS: Unlike DSA, VasoCT is an excellent tool to assess WEB deployment and positioning. In our experience, it allowed a precise evaluation of the WEB sizing and its relation to the parent vessel. Such information very likely enhances the ability to safely use this device, avoiding potential thromboembolic events in cases of protrusion in the parent arteries.

ABBREVIATIONS: WEB $=$ Woven EndoBridge Embolization System

$\mathrm{T}$ he Woven EndoBridge Embolization System (WEB; Sequent Medical, Aliso Viejo, California) is a 2-compartment intrasaccular flow disrupter, particularly dedicated to the treatment of intracranial wide-neck bifurcation aneurysms. This device has an attenuated microbraided mesh structure constructed from a large number of nitinol wires (ranging from 19 to 38 microns). This mesh is almost not visible with standard DSA because of the small diameter of nitinol wires, so 3 platinum markers represent the proximal, middle, and distal parts of inner and outer braids (Fig 1). ${ }^{1,2}$ The first clinical results suggest a high feasibility of the treatment with acceptable morbidity and mortality. ${ }^{3-5}$

Flat panel detector CT has proved to be a very accurate tool to assess the structure and deployment of intracranial stents. ${ }^{6,7}$ VasoCT (Philips Healthcare, Best, the Netherlands) provides simultaneous detailed visualization of neurovascular stents and parent arteries. ${ }^{8,9}$

Received October 9, 2013; accepted after revision November 25.

From the Department of Interventional Neuroradiology (J.C., C.M., H.N., L.I., N.B., J.M., L.S.), Beaujon Medical Centre, Clichy, France; and Interventional X-Ray Innovation (D.R.), Philips Healthcare, Best, the Netherlands.

Please address correspondence to Jildaz Caroff, MD, Beaujon Medical Centre, 100, Boulevard Général Leclerc, 92110 Clichy, France; e-mail: Jildaz.caroff@gmail.com

http://dx.doi.org/10.3174/ajnr.A3860
We present here our experience using VasoCT as a systematic method to control WEB deployment before and after its detachment.

\section{MATERIALS AND METHODS \\ Population}

Between March 2012 and July 2013, twelve patients were treated for 13 intracranial aneurysms with the WEB device in our hospi-
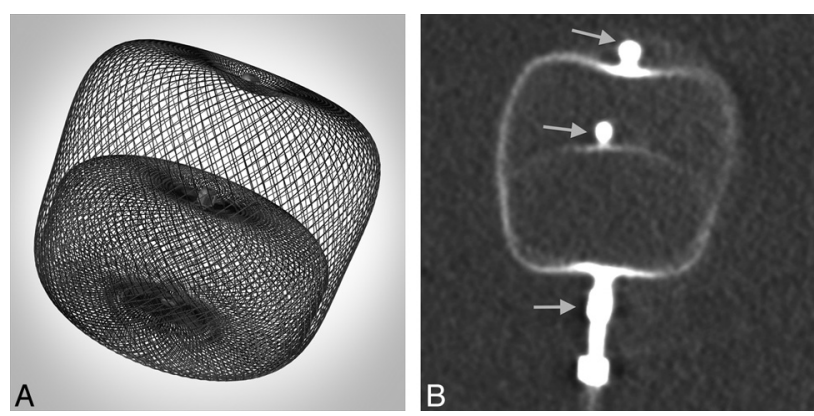

FIG 1. A, Photograph of the WEB device. $B$, Corresponding nonenhanced VasoCT clearly depicts the 2 different compartments and the 3 markers (arrows). 
tal. All subjects gave informed consent for the treatment. There were 10 women and 2 men with a mean age of 52 years (range, $44-55$ years).

\section{Treatment Technique}

Endovascular treatment was performed by using a biplane flat panel angiographic system (Allura Xper 20/10; Philips Healthcare). Endovascular procedures were performed with the patient under general anesthesia and systemic heparinization.

A guiding catheter, FargoMax 6F (Balt, Montmorency,

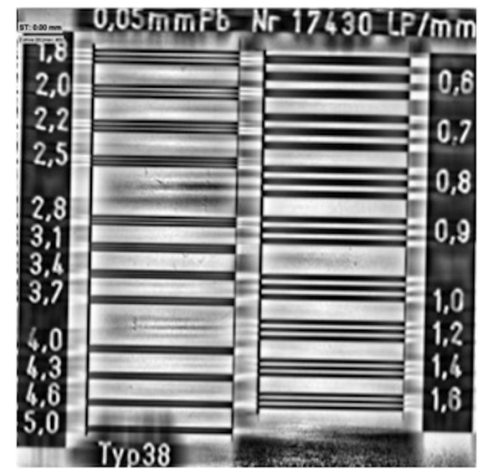

FIG 2. Central section of the VasoCT conebeam reconstruction of a line-pair phantom. The numbers at the left and right side indicate the line pairs per millimeter.
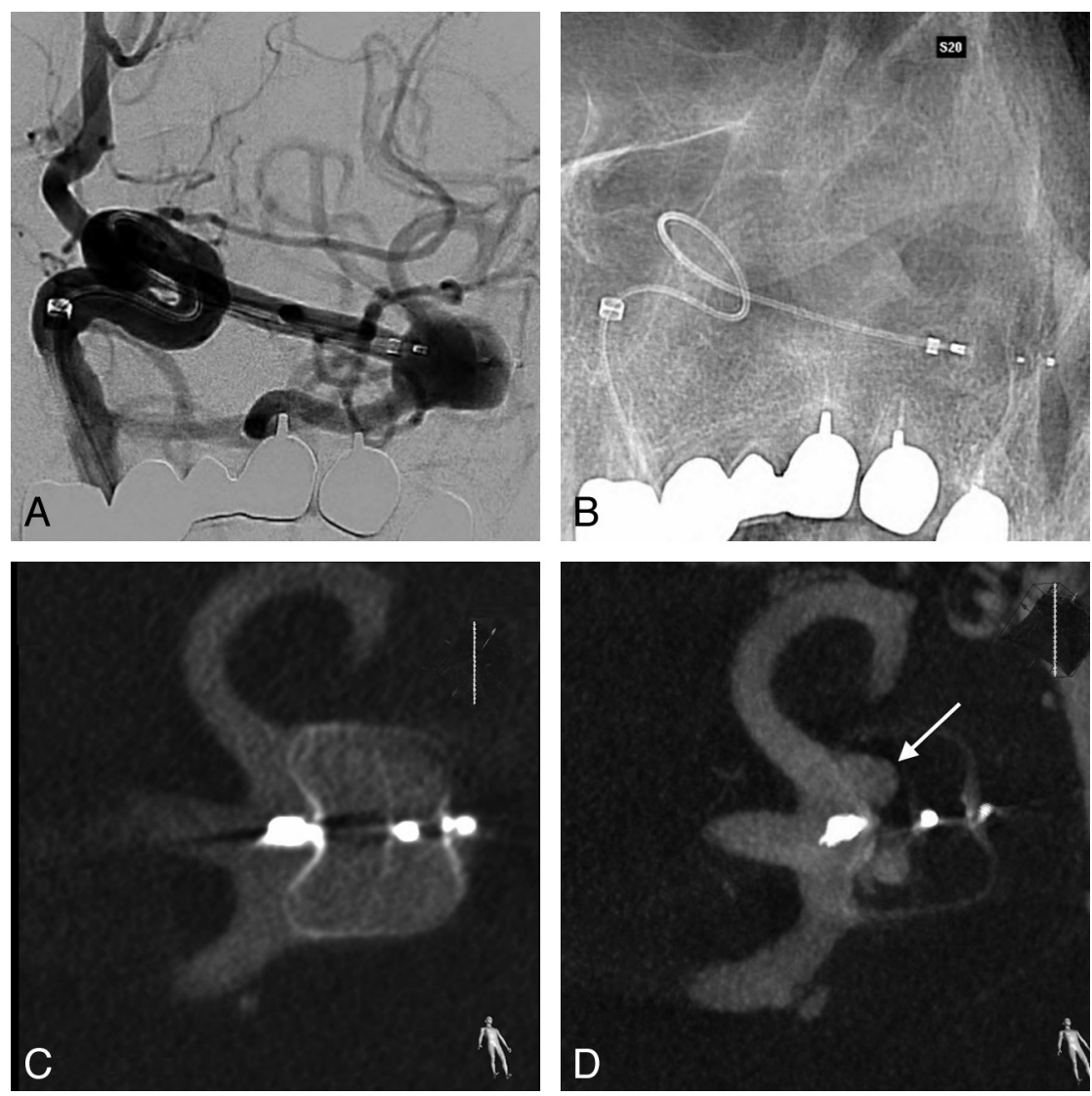

FIG 3. A, DSA before detachment of a WEB positioned in a left MCA bifurcation aneurysm. It is impossible to depict any protrusion of the device in the parent artery because only the 3 markers are seen and the mesh is almost not visible. $B$, Corresponding unsubstracted image. $C$, VasoCT confirms correct positioning of the WEB without any protrusion. $D$, Three-month control VasoCT shows residual flow in the proximal compartment (arrow).
France) was advanced in the internal carotid or vertebral artery. To deliver the WEB, we used different microcatheters and placed them at the base of the aneurysmal sac: Rebar 27 (Covidien, Irvine, California), DAC 38 (Concentric Medical, Mountain View, California), Headway 27 (MicroVention, Tustin, California), and Via 27 or 33 (Sequent Medical). The microcatheter was chosen according to the WEB size. The WEB size choice was based on 3D rotational angiography performed during treatment.

Control DSA and VasoCT were performed before delivery. If the position was not satisfactory, the WEB was resheathed and repositioned. If the sizing was not adequate, the WEB was removed and another device was used. At the end of the procedure, both DSA and VasoCT were repeated after detachment of the WEB.

\section{High-Resolution Flat Panel Detector CT}

For high-spatial-resolution imaging of intravascular devices and their parent vessel lumen, a dedicated iodine contrast-enhanced conebeam CT protocol was developed (VasoCT). It is acquired by using a neuroangiographic x-ray C-arm (Allura Xper FD20; Philips Healthcare) equipped with a cesium iodide-amorphous silicon flat panel detector. The sensor area of the flat detector measures approximately $30 \times 40 \mathrm{~cm}$ and consists of $1920 \times 2480$ pixels. The conebeam acquisition consists of a rotational trajectory over a $200^{\circ}$ arc while acquiring 620 projection images at a fixed source-todetector distance of $1195 \mathrm{~mm}$. The objects of interest should be positioned in the center of the rotation, $810 \mathrm{~mm}$ from the source. The $\mathrm{x}$-ray tube voltage is set to $80 \mathrm{kV}$; the focal spot, to $0.4 \mathrm{~mm}$, while no copper filter is used. The associated radiation dose ranges from 45 to $49 \mathrm{mGy}$ CT dose index. The imaged detector area is fixed to a diameter of $22 \mathrm{~cm}$, which enables an unbinned pixel size of $0.154 \mathrm{~mm}$, allowing very high-spatialresolution $3 \mathrm{D}$ reconstructions. The $3 \mathrm{D}$ reconstruction is obtained by using the Feldkamp-Davis-Kress method. ${ }^{10}$ Preprocessing steps of the projection images include gain correction, scatter correction, water beam-hardening correction, and Parker weighting. The WEB VasoCT was reconstructed with a $512^{3}$ matrix covering a cubic FOV of 34.44 $\mathrm{mm}$ in each dimension.

Figure 2 shows a VasoCT reconstruction of a line-pair phantom. As can be seen, up to 4.6 line pairs per millimeter (lp/mm) can be distinguished, which correspond to an intrinsic resolution of $0.109 \mathrm{~mm}$ according to the following formula: Resolution Intrinsic Millimeter $=0.5[\mathrm{lp} / \mathrm{mm}]$.

The spatial resolution is substantially higher than the resolutions reported for 

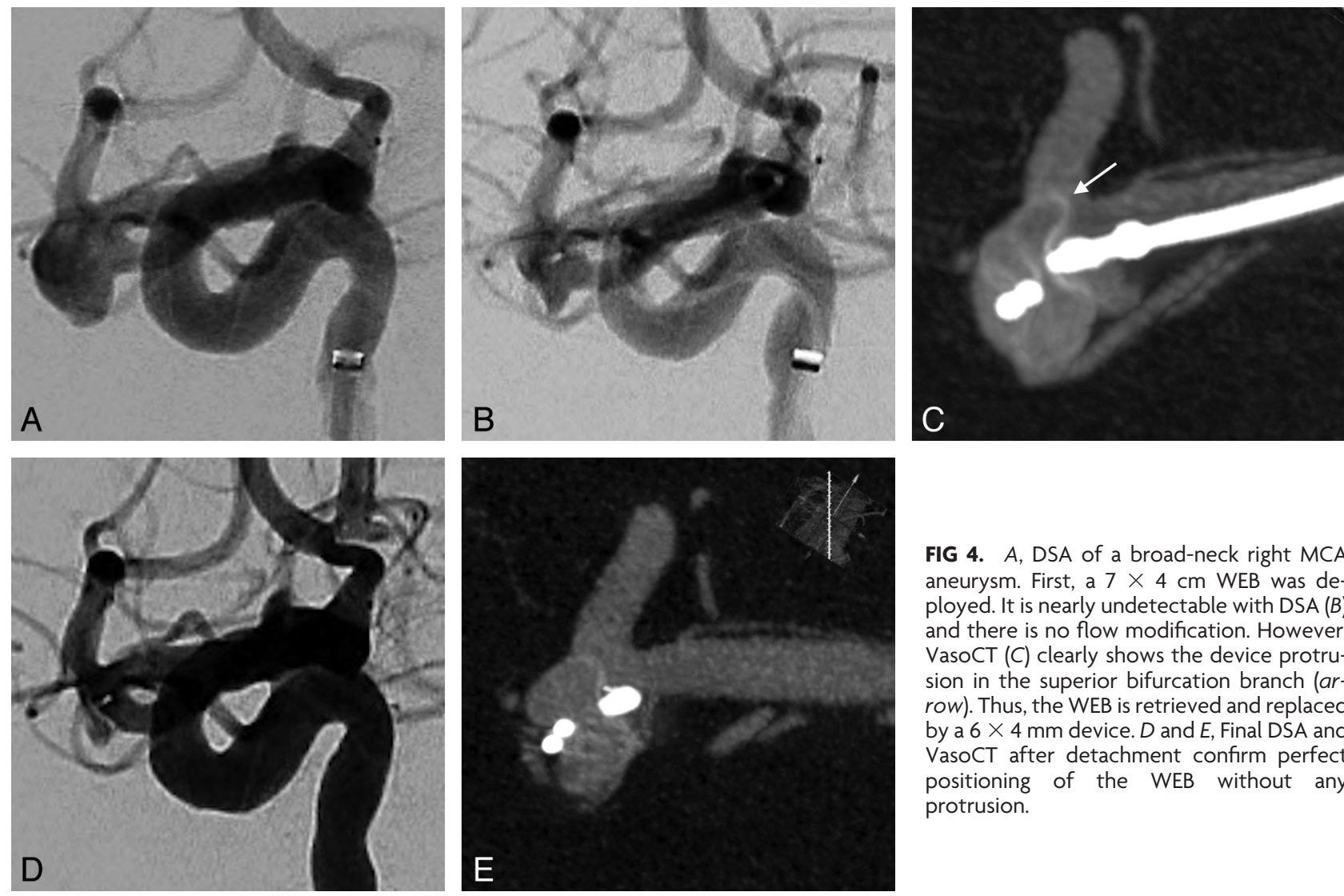

FIG 4. A, DSA of a broad-neck right MCA aneurysm. First, a $7 \times 4 \mathrm{~cm}$ WEB was deployed. It is nearly undetectable with DSA (B) and there is no flow modification. However, VasoCT (C) clearly shows the device protrusion in the superior bifurcation branch (arrow). Thus, the WEB is retrieved and replaced by a $6 \times 4 \mathrm{~mm}$ device. $D$ and $E$, Final DSA and VasoCT after detachment confirm perfect positioning of the WEB without any protrusion.
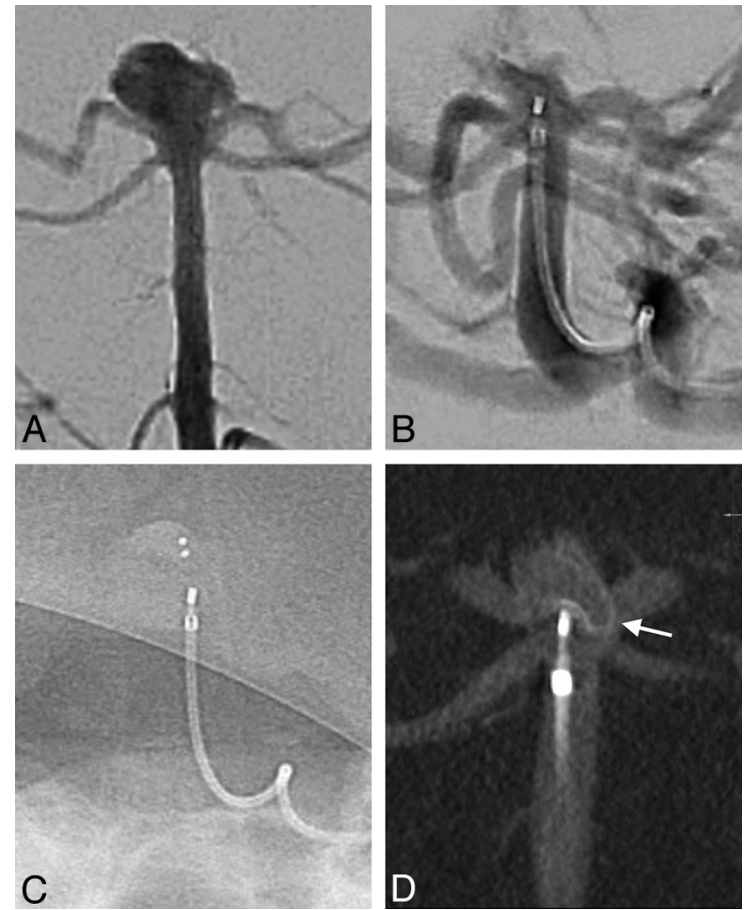

FIG 5. A, DSA anteroposterior view of a basilar tip aneurysm. $B$, Control angiogram and corresponding unsubtracted view $(C)$ prior to detachment of the WEB. Some contrast agent stagnation is visible in the second layer. It is impossible to analyze the good positioning of the device; no flow abnormality is depicted in the left P1 segment. $D$, VasoCT clearly shows the protrusion in the left PI segment. The WEB was then retrieved and replaced by a smaller one. multisection CT. For 64-section CT, a resolution of approximately $1.5 \mathrm{lp} / \mathrm{mm}$ has been reported. ${ }^{11}$ The high spatial resolution of VasoCT allows imaging the stent struts of intracranial nitinol stents. ${ }^{9}$ To visualize the vessel lumen and the intravascular devices simultaneously, we used a diluted iodine contrast agent with 20\% iodixanol (Visipaque 270; GE Healthcare, Mississauga, Ontario, Canada) and $80 \%$ saline solution. The total volume used was $90 \mathrm{~mL}$ at the injection rate of $3 \mathrm{~mL} / \mathrm{s}$. In case of streaking artifacts caused by nearby metal (eg, coils), the image quality can be improved by applying a metal-artifactsreduction algorithm in a second-pass reconstruction. ${ }^{12}$ Data were analyzed with MPR and MIP reconstructions.

\section{Quality Assessment}

VasoCT scans were reviewed in consensus by 2 interventional neuroradiologists. For every case, the quality of visualization of the WEB and the parent vessels was scored in ordered scales from 1 to 3 . A score of 1 indicated that visualization was insufficient for evaluation; a score of 2 was given if the visualization was good; and a score of 3 , if excellent. Metal artifacts from the WEB device and microcatheter, if present, were also evaluated. A score of 3 was given when no artifacts were depicted; a score of 2, if mild artifacts were observed. A score of 1 indicated severe artifacts, making evaluation of the WEB positioning impossible.

Those scores were added to obtain a global quality score on a 9-point subjective scale. Global VasoCT quality scores were compared with WEB dimensions by using the Pearson correlation coefficient.

AJNR Am J Neuroradiol 35:1353-57 Jul 2014 www.ajnr.org 

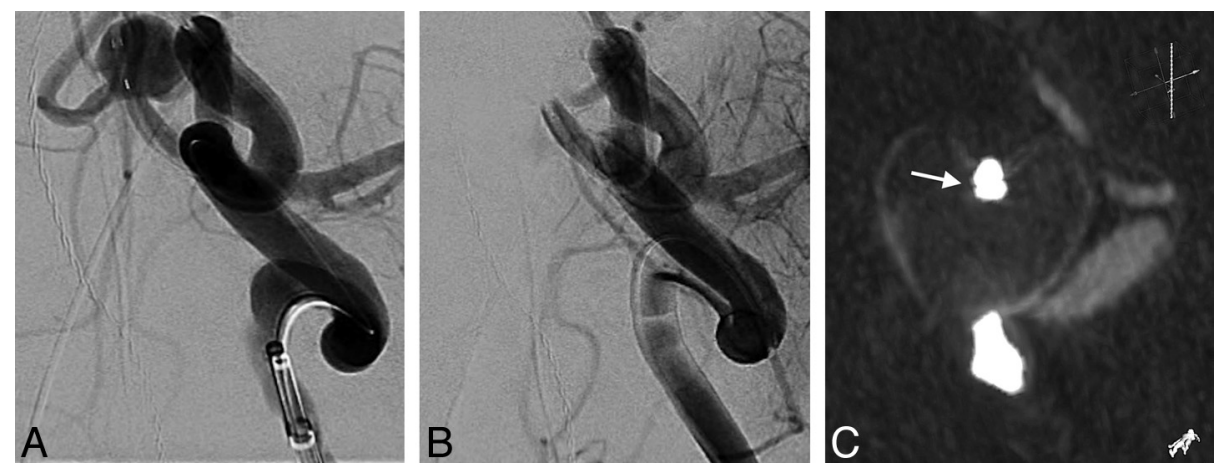

FIG 6. A, DSA working projection of a large-neck anterior communicating artery aneurysm. $B$, After placement of a $9 \times 7 \mathrm{~mm}$ WEB, the control DSA shows the occlusion of the right A2 segment and a narrowing of the left A2. C, VasoCT confirms the occlusion and that the WEB is not properly deployed. It is in a heart-shaped configuration with a distal trough; the 2 distal markers are attached (arrow). This "heart sign" depicts an inappropriate width of the WEB regarding aneurysm dimensions. It was then replaced by a correctly deployed $7 \times 6 \mathrm{~cm}$ WEB.

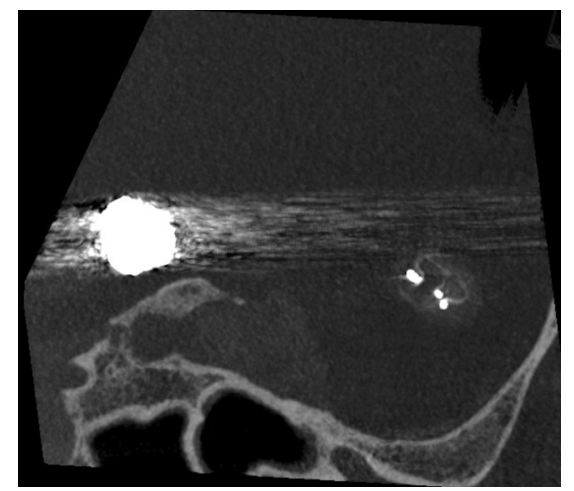

FIG 7. Nonenhanced VasoCT. It is very important to consider the acquisition plane to avoid the projection of any metal artifacts over the region of interest. Here, the angulation of the head of the patient was modified so that coil artifacts from a previously treated basilar tip aneurysm do not superimpose on the MCA bifurcation.

Additionally, reviewers had to evaluate whether any protrusion, clotting, vessel occlusion, or residual neck was present.

\section{RESULTS}

Most treated aneurysms were unruptured (3 ruptured cases), with an unfavorable wide-neck anatomy (mean dome/neck ratio $=$ 1:1). The mean aneurysm size was $7.3 \mathrm{~mm}$ (range, 5-11 mm).

WEB placement was not possible in 1 case because of the unavailability of an appropriate-sized device. Device protrusion was clearly detected with VasoCT in 5 cases, leading to WEB repositioning or size substitution (Figs 3 and 4 ). The average number of WEBs used to obtain good sizing was 1.3 per procedure.

The total number of VasoCT scans reviewed was 31, with an average number of 2.4 VasoCT scans performed per procedure. The mean quality score was $6.6 \pm 1.4$ (range, $4-9$ ). Visualization of the WEB and/or the parent vessel was insufficient for evaluation in 3 cases of VasoCT ( $9 \%)$.

Quality score was significantly and inversely correlated to the WEB height $(r=0.78, P<.0001)$.

\section{DISCUSSION}

The visibility of the WEB is very poor with conventional angiography (Fig 5). Unlike DSA, VasoCT offers a very good visualization of the microbraided mesh structure and shape of the WEB device. It allows a precise study of its relationship with the parent arteries. This is fundamental before detachment of this completely retrievable device to avoid potential thromboembolic complications due to protrusion (Fig 6).

We have encountered some differences in VasoCT image quality. Some features seem to be significantly influencing the visibility of the WEB. The device should always be placed in the isocenter of the acquired volume. All movements of the patient have to be limited; therefore, apnea is required during acquisition. No geometric disturbances should occur; these can be seen when the $\mathrm{C}$-arm hits the infusion stand or table. High-attenuating items need to be avoided in the reconstruction plane of the WEB by positioning the head of the patient so that coils or clip streak artifacts appear in another plane (Fig 7). Metal artifacts reduction may be useful in some cases, especially when WEB-marker artifacts are projected over the parent artery (ie, MCA bifurcation aneurysms) (Fig 8).

WEB markers made of iridium and platinum alloy generate significant metal artifacts (unlike nitinol mesh). We have noticed that distal markers of the delivery microcatheter, made with the same iridium and platinum alloy, also induce artifacts; they can be avoided by retrieving the catheter proximally in the parent artery.

The quality score was inversely correlated to the WEB height. This observation can be explained first because the diameter of nitinol wires is variable (ie, smaller for the smallest WEB). Thus, the largest WEB sizes are more radiodense and easily assessed. This feature should encourage manufacturers to increase the visibility of the smaller devices. Second, the distance between the markers is small for the small WEB, leading to overlapping metal streak artifacts that are summed, deteriorating the visualization of the parent arteries.

We advise a systematic calibration of the angiographic suite prior to each acquisition. When the system is used for a long time with a reduced FOV (as is usual during a procedure), then the pixels that have been irradiated within this image format have a different response than the ones that were not irradiated. The calibration corrects this phenomenon, allowing an optimal image quality.

Some authors report the use of $3 \mathrm{D}$ rotational angiography to control WEB positioning. ${ }^{5}$ There are similarities and differences 

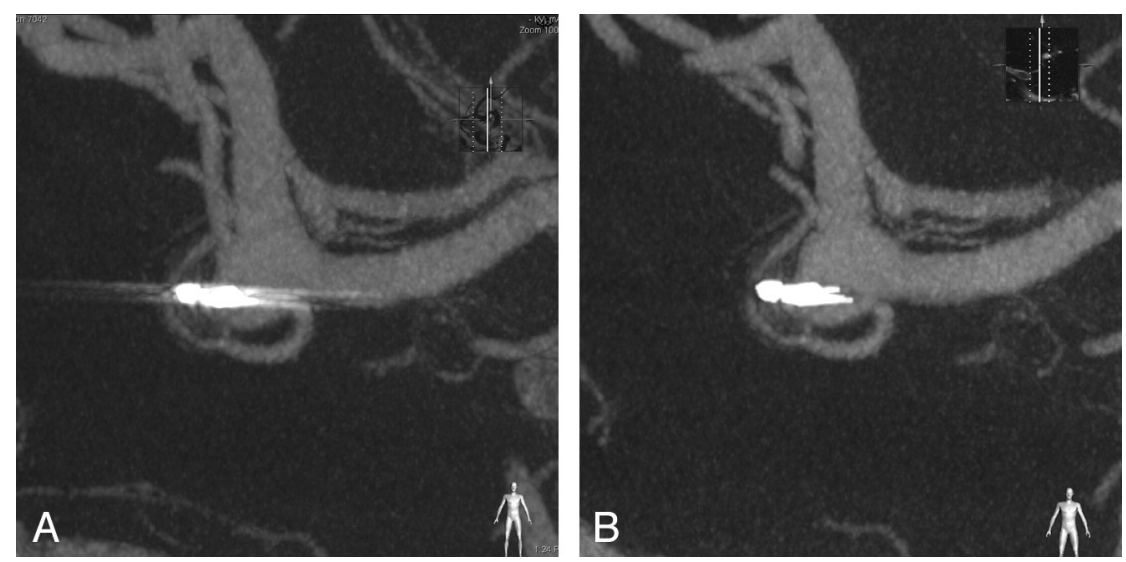

FIG 8. A, VasoCT of an MCA aneurysm treated with a WEB. Marker artifacts are projecting over the parent vessels. $B$, A new reconstruction with a metal-artifacts-reduction algorithm clearly improves the image quality and the visualization of the bifurcation.

between VasoCT and 3D rotational angiography. Both protocols are optimized for visualization of iodine contrast; hence, both use approximately $80 \mathrm{kV}$ for the $\mathrm{x}$-ray tube and both are optimized for high-resolution reconstructions (either volume-rendering or multiplanar mode). While VasoCT is meant for small contrast differences (with diluted contrast medium), 3D rotational angiography is meant for larger contrast differences (with pure contrast medium). To achieve the high contrast resolution, VasoCT acquires 620 images, whereas 3D rotational angiography records 120 images in the rotation. As a result, the VasoCT acquisition takes longer (20 versus 4 seconds); and because of the larger number of images, the reconstruction times for VasoCT are also longer (approximately 26 seconds versus 5 seconds for $256^{3}$ ). Because of its superior contrast resolution, VasoCT is the most appropriate technique for use with the WEB device.

VasoCT $\mathrm{x}$-ray skin dose calculated from simulations amounts to $0.89 \mathrm{mSv}$, and the effective dose is $0.51 \mathrm{mSv}$. For comparison, the standard brain CT effective dose average is $1.5 \mathrm{mSv}{ }^{13}$

While VasoCT appears essential during endovascular treatment, it is also very useful during follow-up. It can be performed with the patient under local anesthesia, and it allows the assessment of any residual flow inside the 2 separate layers (Fig 3).

\section{CONCLUSIONS}

Unlike DSA, VasoCT is an excellent tool to assess WEB deployment and positioning. In our experience, it allows a precise evaluation of the WEB sizing and its relation to the parent vessel. Such information likely enhances the ability to use this device safely, avoiding a potential thromboembolic event in cases of protrusion in the parent arteries.

Disclosures: Daniel Ruijters—UNRELATED: Employment: I am a Philips Healthcare employee. Jacques Moret—UNRELATED: Consultancy: Covidien, MicroVention.

\section{REFERENCES}

1. Ding YH, Lewis DA, Kadirvel R, et al. The Woven EndoBridge: a new aneurysm occlusion device. AJNR Am J Neuroradiol 2011;32:607-11

2. Klisch J, Sychra V, Strasilla C, et al. The Woven EndoBridge cerebral aneurysm embolization device (WEB II): initial clinical experience. Neuroradiology 2011;53:599-607

3. Pierot L, Liebig T, Sychra V, et al. Intrasaccular flow-disruption treatment of intracranial aneurysms: preliminary results of a multicenter clinical study. AJNR Am J Neuroradiol 2012;33:1232-38

4. Lubicz B, Mine B, Collignon L, et al. WEB device for endovascular treatment of wide-neck bifurcation aneurysms. AJNR Am J Neuroradiol 2013;34:1209-14

5. Pierot L, Klisch J, Cognard C, et al. Endovascular WEB flow disruption in middle cerebral artery aneurysms: preliminary feasibility, clinical, and anatomical results in a multicenter study. Neurosurgery 2013;73:27-35, discussion 34-35

6. Richter G, Engelhorn T, Struffert T, et al. Flat panel detector angiographic CT for stent-assisted coil embolization of broad-based cerebral aneurysms. AJNR Am J Neuroradiol 2007;28:1902-08

7. Benndorf G, Strother CM, Claus B, et al. Angiographic CT in cerebrovascular stenting. AJNR Am J Neuroradiol 2005;26:1813-18

8. Patel NV, Gounis MJ, Wakhloo AK, et al. Contrast-enhanced angiographic cone-beam CT of cerebrovascular stents: experimental optimization and clinical application. AJNR Am J Neuroradiol 2011;32:137-44

9. Snoeren RM, Söderman M, Kroon JN, et al. High-resolution 3D Xray imaging of intracranial nitinol stents. Neuroradiology 2012; 54:155-62

10. Feldkamp L, Davis L, Kress J. Practical cone-beam algorithm. J Opt Soc Am A Opt Image Sci Vis 1984;1:612-19

11. Flohr T, Stierstorfer K, Raupach R, et al. Performance evaluation of a 64-slice CT system with z-flying focal spot. Rofo 2004;176:1803-10

12. van der Bom IM, Hou SY, Puri AS, et al. Reduction of coil mass artifacts in high-resolution flat detector conebeam CT of cerebral stent-assisted coiling. AJNR Am J Neuroradiol 2013;34:2163-70

13. van der Molen AJ, Schilham A, Stoop P, et al. A national survey on radiation dose in CT in the Netherlands. Insights Imaging 2013;4: 383-90 